\title{
Neoplasias do sistema nervoso central de caninos e felinos: estudo de 20 anos
}

\author{
Neoplasms of the canine and feline central nervous system: 20-year study \\ Neoplasias del sistema nervioso central canino y felino: estudio de 20 años
}

\section{Resumo}

O objetivo desse estudo foi avaliar os aspectos epidemiológicos e patológicos de neoplasias neurológicas em caninos e felinos encaminhados ao Laboratório Regional de Diagnóstico da Faculdade de Veterinária da Universidade Federal de Pelotas (LRD/FV/UFPel) em um período de 20 anos. Foram revisados os protocolos de necropsia de caninos e felinos encaminhados ao LRD/FV/UFPel de janeiro de 2000 a dezembro de 2020, sendo selecionados os casos com diagnóstico de neoplasia neurológica primária e secundária. Destes foram analisadas as informações referentes à idade, sexo, raça, sinais clínicos, achados macroscópicos e histopatológicos. No período estudado foram diagnosticados 35 casos de neoplasias no sistema nervoso central de caninos e seis em felinos. Em ambas as espécies, as neoplasias se mostraram mais frequentes nas fêmeas, animais sem raça definida e com mais de cinco anos de idade, sendo os tumores secundários os mais prevalentes. A região neuroanatômica mais acometida nos caninos foi a tálamocortical e nos felinos a medula espinhal. Nos cães o sinal clínico neurológico mais frequente foi convulsão e nos gatos não houve maior ocorrência de nenhum sinal clínico. A ausência de sinais neurológicos foi observada em 37,5\% dos caninos e em 50\% dos felinos. As principais neoplasias relacionadas a causa de morte de cães e gatos, neste estudo, foram as secundárias, sendo a metástase de carcinoma de mama o principal diagnóstico, podendo ainda, em muitos casos não serem observados sinais clínicos neurológicos.

Palavras-chave: Metástase neurológica; Meningioma; Carcinoma mamário; Cães; Gatos.

\section{Abstract}

The aim of this study was to evaluate the epidemiological and pathological aspects of neurological neoplasms in canines and felines referred to the Laboratório Regional de Diagnóstico of the Faculdade de Veterinária of the Universidade Federal de Pelotas (LRD/FV/UFPel) over a period of 20 years. The protocols for necropsy of canines and felines sent to the LRD/FV/UFPel from January 2000 to December 2020 were reviewed, and cases with a 
diagnosis of primary and secondary neurological neoplasia were selected. From these, information regarding age, sex, race, clinical signs, macroscopic and histopathological findings were analyzed. During the study period, 35 cases of neoplasms in the central nervous system of canines and six in felines were diagnosed. In both species, neoplasms were more frequent in females, mixed breed animals over five years of age, with secondary tumors being the most prevalent. The most affected neuroanatomical region in canines was the thalamus-cortical region and in felines the spinal cord. In dogs, the most frequent neurological clinical sign was seizure and in cats there was no higher occurrence of any clinical sign. The absence of neurological signs was observed in $37.5 \%$ of canines and $50 \%$ of felines. The main neoplasms related to the cause of death of dogs and cats in this study were the secondary ones, with metastasis from mammary carcinoma being the main diagnosis, and in many cases neurological clinical signs may not be observed.

Keywords: Neurological metastasis; Meningioma; Mammary carcinoma; Dogs; Cats.

\section{Resumen}

El objetivo de este estudio fue evaluar los aspectos epidemiológicos y patológicos de las neoplasias neurológicas en caninos y felinos remitidos al Laboratório Regional de Diagnóstico de la Faculdade de Veterinária de la Universidade Federal de Pelotas (LRD/FV/UFPel) durante un período de 20 años. Se revisaron los protocolos de necropsia de caninos y felinos enviados al LRD/FV/UFPel desde enero de 2000 a diciembre de 2020 y se seleccionaron casos con diagnóstico de neoplasia neurológica primaria y secundaria. A partir de estos, se analizó información sobre edad, sexo, raza, signos clínicos, hallazgos macroscópicos e histopatológicos. Durante el período de estudio se diagnosticaron 35 casos de neoplasias en el sistema nervioso central de caninos y seis en felinos. En ambas especies, las neoplasias fueron más frecuentes en hembras, mestizos mayores de cinco años, siendo los tumores secundarios los más prevalentes. La región neuroanatómica más afectada en caninos fue la región tálamo-cortical y en felinos la médula espinal. En los perros, el signo clínico neurológico más frecuente fueron las convulsiones y en los gatos no hubo mayor ocurrencia de ningún signo clínico. La ausencia de signos neurológicos se observó en el 37,5\% de los caninos y en el $50 \%$ de los felinos. Las principales neoplasias relacionadas con la causa de muerte de perros y gatos en este estudio fueron las secundarias, siendo la metástasis de carcinoma de mama el diagnóstico principal, pudiendo en muchos casos no observarse signos clínicos neurológicos.

Palabras clave: Metástasis neurológica; Meningioma; Carcinoma de mama; Perros; Gatos.

\section{Introdução}

As doenças do sistema nervoso central (SNC) são responsáveis por 7\% das causas de morte e eutanásia em caninos e $10 \%$ em felinos, variando de acordo com as populações estudadas (Chaves et al., 2018b; Frade et al., 2018). Dentre as patologias do SNC as neoplasias representam 5,9\% das afecções neurológicas de caninos e 10,3\% em felinos, sendo consideradas a principal afecção neurológica em animais de meia idade e idosos, podendo ser primárias ou secundárias a metástase ou expansão tumoral (Santos et al., 2012; Horta et al., 2013; Cantile et al., 2016; Higgins et al., 2017; Chaves et al., 2018b; Frade et al., 2018). Alterações encefálicas secundárias como inflamação, necrose, hidrocefalia obstrutiva, edema e herniação cerebral, podem ser observadas e influenciam diretamente no quadro clínico de animais com neoplasias neurológicas (Fraga et al., 2014; Frank et al., 2020).

Neoplasias neurológicas primárias, na maioria dos casos, são tumores focais, histologicamente benignos, raramente com manifestações sistêmicas e metástases (Coates et al., 2010; Horta et al., 2013; Prates et al., 2018; Zamboni et al., 2020). Já as neoplasias secundárias apresentam-se normalmente como lesões multifocais decorrentes de metástase por via hematógena e/ou linfática, com posterior disseminação pelo líquido cefalorraquidiano. A presença de neoplasia secundária indica avançado estágio da doença, com prognóstico desfavorável (Coates et al., 2010; Horta et al., 2013; Mcentee \& Dewey, 2013).

Os sinais clínicos de neoplasia neurológica dependem da localização, expansão e taxa de crescimento do tumor, bem como, das alterações encefálicas secundárias presentes (Horta et al., 2013; Fraga et al., 2014). As crises epilépticas e as alterações comportamentais são os sinais clínicos mais frequentes, podendo ser indicativos precoces de neoplasia no SNC (Snyder et al., 2006; Horta et al., 2013; Chaves et al., 2018a). Os sinais neurológicos e exames de imagem podem indicar a presença de neoplasia no tecido nervoso, porém o diagnóstico definitivo é estabelecido apenas pela análise histopatológica de amostras de biópsia ou necropsia (Santos et al., 2012; Besalti et al., 2016; Prates et al., 2018).

A falta de informações clínicas e o alto custo de exames complementares tornam muitas vezes o diagnóstico de 
neoplasia neurológica um desafio (Andrade et al., 2019; Belluco et al., 2019). Tendo em vista a importância das neoplasias neurológicas na clínica de pequenos animais, o objetivo deste estudo foi avaliar os aspectos epidemiológicos e patológicos das neoplasias do SNC de caninos e felinos diagnosticadas no Laboratório Regional de Diagnóstico, da Faculdade de Veterinária, da Universidade Federal de Pelotas (LRD/FV/UFPel), em um período de 20 anos.

\section{Metodologia}

Foi realizado um levantamento no banco de dados do LRD/FV/UFPel dos protocolos de necropsia de caninos e felinos com diagnóstico de neoplasias do SNC, no período de janeiro de 2000 a dezembro de 2020. Dos protocolos de necropsia foram analisados os dados epidemiológicos dos casos, obtendo-se informações referentes à idade, sexo, raça, sinais clínicos, achados macroscópicos e microscópicos, bem como, o diagnóstico que constava no protocolo original dos arquivos.

Os animais foram agrupados em três categorias de acordo com a faixa etária. Animais com até um ano de idade foram classificados como filhotes, de um a dez anos como adultos e com mais de dez anos como idosos. Em relação a raça foram classificados como sem raça definida (SRD) e com raça definida (CRD). Nos casos sem informações referentes a idade, sexo e/ou raça foram agrupados como não informados (NI).

As neoplasias foram classificadas como primárias quando apresentavam origem no tecido nervoso (células da glia e neurônios), nas células de revestimento (interno e externo) do encéfalo (meninge e epêndima) ou no plexo coroide. Como secundárias quando decorrentes de metástases ou expansão tumoral para o SNC. A localização dos tumores foi estabelecida de acordo com os sinais neurológicos, conforme descrito por Santos et al. (2012) e achados macroscópicos. As lesões foram classificadas em quatro regiões neuroanatômicas: tálamo-córtex, tronco-encefálico, cerebelo e medula espinhal. As lesões medulares foram separadas em três regiões: cervical, torácica e lombar. Os casos que apresentavam o envolvimento de mais de uma região anatômica foram classificados como multifocais, e os sem histórico clínico e descrição macroscópica como não informados (NI).

\section{Resultados}

No período de 2000 a 2020 foram recebidos no LRD/FV/UFPel 8003 amostras de caninos e 1518 de felinos, sendo $447 / 8003(5,6 \%)$ e 127/1518 (8,4\%) diagnosticados como doenças neurológicas em ambas as espécies. Das patologias do SNC diagnosticadas, as neoplasias corresponderam a 7,8\% (35/447) nos caninos e 4,7\% (6/127) nos felinos. Quanto ao perfil dos caninos acometidos por neoplasias neurológicas 22/35 (62,9\%) eram fêmeas e 13/35 (37,1\%) machos, 19/35 (54,3\%) eram idosos, $13 / 35(37,1 \%)$ adultos e em três $(8,6 \%)$ casos a idade não foi informada. Em relação a raça 13/35 (37,1\%) cães eram SRD e 22/35 (62,9\%) CRD, sendo o Cocker a raça mais acometida, com quatro casos. Nos felinos 5/6 $(83,3 \%)$ animais eram fêmeas e 1/6 (16,7\%) era macho; $3 / 6(50 \%)$ eram adultos, $2 / 6$ idosos (33,3\%) e 1/6 jovem (16,7\%); 4/6 (66,7\%) eram SRD e 2/6 (33,3\%) da raça Persa. Na Tabela 1 está exemplificado o perfil dos caninos e felinos acometidos por neoplasias neurológicas. 
Tabela 1 - Perfil de caninos e felinos diagnosticados com neoplasias neurológicas no LRD/FV/UFPel, no período de 2000 a 2020 .

\begin{tabular}{lcllc}
\hline Espécie & $\begin{array}{c}\text { Número total de } \\
\text { casos (n) }\end{array}$ & Raça (n) & Sexo (n) & Idade (n) \\
\hline Caninos & 35 & SRD (13) & Fêmea (22) & Adulto (13) \\
& & CRD (22) & Idoso (19) \\
& & & Fêmea (13) (3) & Jovem (1) \\
\cline { 2 - 5 } Felinos & 6 & SRD (4) & Macho (1) & Adulto (3) \\
& & CRD (2) & & Idoso (2) \\
\hline
\end{tabular}

NI: Não informado. Fonte: Autores.

Sinais clínicos neurológicos foram observados em 23/35 caninos e em 3/6 felinos. Nos caninos os sinais clínicos observados foram convulsão (7/23), paralisia (6/23), ataxia (4/23), incoordenação (4/23), alteração de comportamento (3/23), cegueira (3/23), nistagmo (2/23), perda da propriocepção (1/23), pressão da cabeça (1/23) e torneio (1/23). Em quatro casos o único sinal clínico observado foi convulsão, sendo três destes casos tumores primários, com diagnóstico de meningioma. Nos felinos os sinais neurológicos apresentados foram incoordenação (1/3), ataxia (1/3), paralisia dos membros posteriores (1/3) e incontinência fecal e urinária (1/3). A ausência de sinais clínicos neurológicos e presença de sinais inespecíficos como apatia, anorexia e emagrecimento foi observado em 12/35 caninos e em 3/6 felinos.

A região tálamo-córtex foi a mais afetada nos caninos (17/35), seguida pelas lesões multifocais (7/35), medula espinhal (4/35) e cerebelo (3/35). Em 3/4 casos de neoplasias medulares foi possível determinar o segmento da medula afetado, sendo um caso de carcinoma tubular na cervical, 1/3 linfoma na torácica e 1/3 carcinoma de glândula hepatóide na porção final da lombar (Figura 2D). Em 4/35 casos não foi possível determinar a localização dos tumores nesta espécie. Nos felinos 3/6 neoplasmas ocorreram na medula espinhal, 1/6 na região tálamo-córtex, 1/6 no cerebelo e 1/6 foi multifocal. Em relação aos segmentos de medula afetados a cervical foi acometida em um caso de carcinoma de tubular e a lombar em dois casos, um fibrossarcoma e um linfoma.

Nos caninos as neoplasias secundárias (Figura 1A, B, C) representaram 25/35 (71,4\%) diagnósticos, sendo as metástases de carcinoma mamário $(8 / 25 ; 32 \%)$ e de hemangiossarcoma $(5 / 25 ; 20 \%)$ (Figura 2C) as de maior ocorrência. Dentre os tumores de mama foram observadas metástases de carcinoma tubular (3/8), túbulo-papilar (2/8), complexo (1/8), sólido (1/8) e anaplásico (1/8) (Figura 2A). Os tumores primários (Figura 1D, E, F e Figura 2E, F) nesta espécie corresponderam 10/35 (28,6\%) casos, sendo o meningioma (7/10; 70\%) o principal diagnóstico. Os subtipos de meningioma diagnosticados foram o psamomatoso (3/7), transicional (3/7) e microcístico (1/7). Nos felinos as neoplasias secundárias, também, foram as mais frequentes $(5 / 6 ; 83,3 \%)$, sendo a metástase de carcinoma de mama $(2 / 5 ; 40 \%)$ a de maior ocorrência. As duas variantes de carcinoma mamário que ocasionaram metástase para o SNC em felinos foram tubular (Figura 2B) e cribriforme. O meningioma fibroso $(1 / 6 ; 16,7 \%)$ foi o único tumor primário diagnosticado em felinos, no período estudado. Nas Tabelas 2 e 3 estão determinados o perfil dos animais acometidos e a origem e localização dos tumores em felinos e caninos respectivamente. 
Figura 1 - Macroscopia de neoplasias neurológicas em caninos. 1A - Metástase de osteossarcoma extraesquelético, substituindo o tecido nervoso na região entre córtex parietal e occipital (seta). 1B - Carcinoma de glândula hepatóide metastático na medula espinhal (seta). 1C - Áreas multifocais, vermelho-enegrecidas, correspondentes à metástase de hemangiossarcoma (setas). 1D - Meningioma focal na região de bulbo olfatótio e córtex frontal (seta). 1E - Lesões multifocais de meningioma no córtex temporal (setas). 1F - Neuroblastoma olfatório infiltrando tecido nervoso na região de córtex frontal (seta).
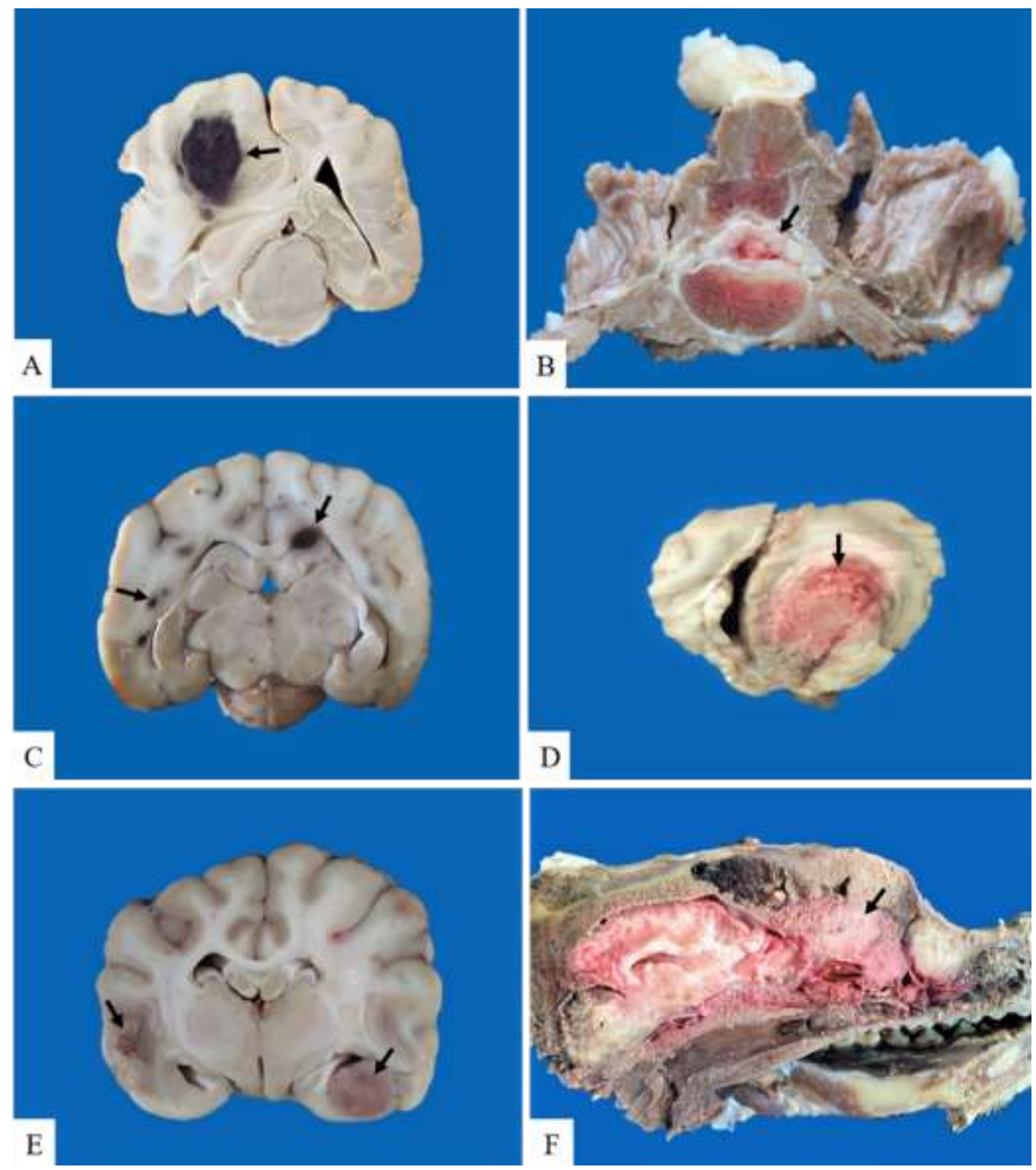

Fonte: Autores.

Tanto em caninos como em felinos em $100 \%$ dos casos de neoplasia mamária com metástases no SNC, também, foram observadas metástases em pulmão e linfonodos. No caso dos hemangiossarcomas em caninos em $80 \%$ dos casos havia também metástase no pulmão, fígado e rim. 
Figura 2 - Microscopia de neoplasias, primárias e secundárias, no sistema nervoso central de caninos e felinos. $1^{\mathrm{a}}-$ Cerebelo de canino com metástase de carcinoma anaplásico de mama (*). HE, obj.40x. 1B - Metástase de carcinoma tubular de mama no encéfalo de felino (*). HE, obj.40x. 1C - Hemangiossarcoma secundário em córtex encefálico de um canino, com grande quantidade de sangue e formação de vasos (setas). HE, obj.4x. 1D - Metástase de carcinoma de glândula hepatóide no canal medular em canino (*), com compressão dos nervos da cauda equina (seta). HE, obj.4x. 1E - Meningioma microcístico no encéfalo de canino (*). HE, obj.10x. 1F - Neuroblastoma em canino, infiltrando córtex frontal (*). HE, obj.4x.
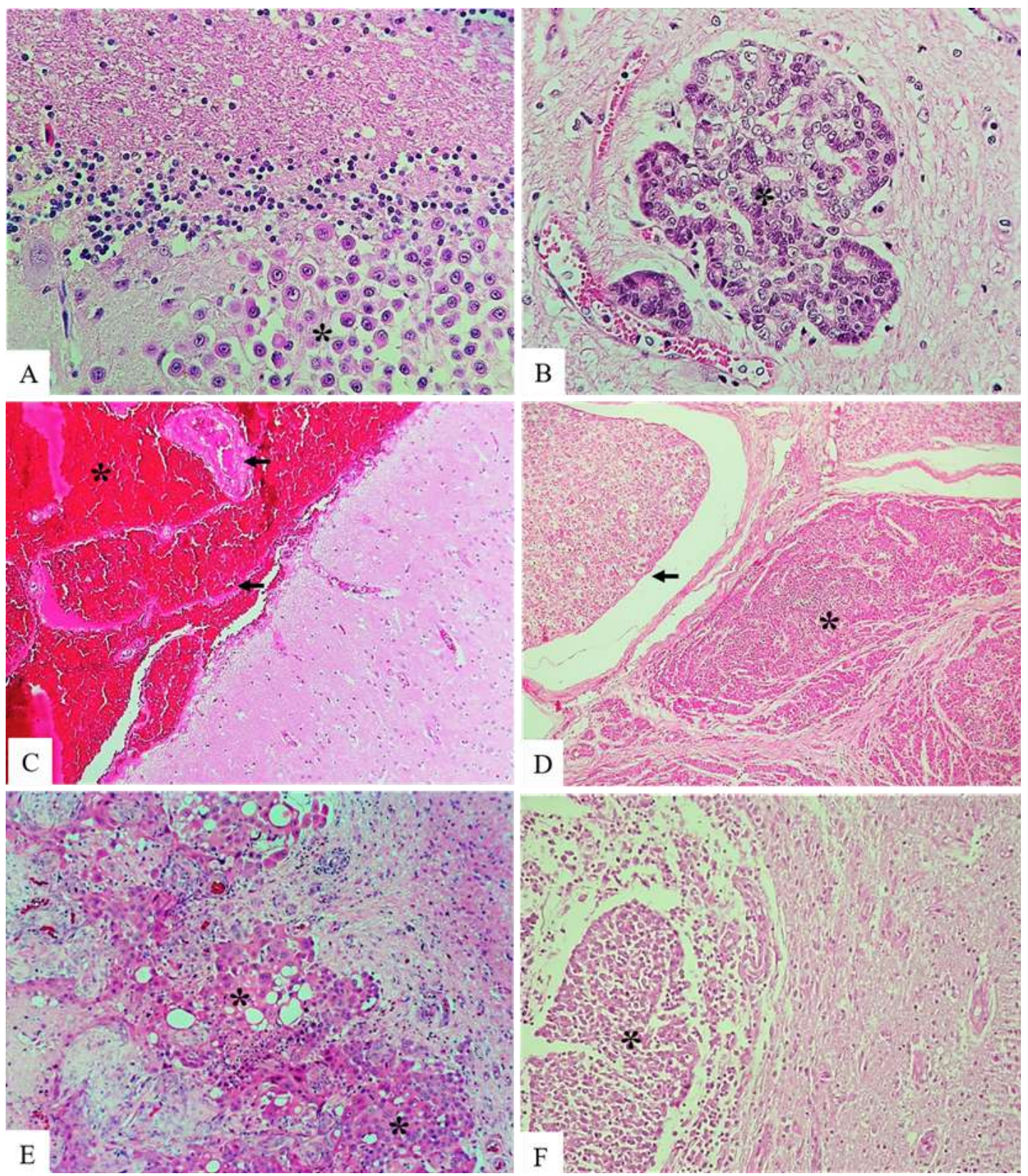

Fonte: Autores. 
Tabela 2 - Classificação do tipo, origem e localização das neoplasias neurológicas dos felinos encaminhados ao LRD/FV/UFPel e determinação do sexo, idade e raça dos animais acometidos.

\begin{tabular}{lcccccc}
\hline \multicolumn{1}{c}{ Diagnóstico } & N & Origem & $\begin{array}{c}\text { Localização } \\
(\mathbf{n})\end{array}$ & $\begin{array}{c}\text { Sexo } \\
(\mathbf{n})\end{array}$ & $\begin{array}{c}\text { Idade média } \\
\text { (anos) }\end{array}$ & Raça (n) \\
\hline Carcinoma de mama & 2 & $\mathrm{~S}$ & $\mathrm{ME}(1)$ & $\mathrm{F}(2)$ & 12,5 & Persa (1) \\
Fibrossarcoma & & & $\mathrm{C}(1)$ & & & $\mathrm{SRD}(1)$ \\
Linfoma multicêntrico & 1 & $\mathrm{~S}$ & $\mathrm{ME}(1)$ & $\mathrm{M}(1)$ & 4 & $\mathrm{SRD}(1)$ \\
Meningioma & 1 & $\mathrm{~S}$ & $\mathrm{ME}(1)$ & $\mathrm{F}(1)$ & 1 & $\mathrm{SRD}(1)$ \\
Mieloma múltiplo & 1 & $\mathrm{P}$ & $\mathrm{TC}(1)$ & $\mathrm{F}(1)$ & 11 & $\mathrm{Persa}(1)$ \\
\hline
\end{tabular}

$\mathrm{P}=$ Primária, $\mathrm{S}=$ secundária, $\mathrm{TC}=$ tálamo córtex, $\mathrm{C}=$ cerebelo, $\mathrm{ME}=$ medula espinhal, $\mathrm{MF}=$ multifocal, $\mathrm{M}=$ macho, F = fêmea, $\mathrm{SRD}=$ sem raça definida. Fonte: Autores.

Tabela 3 - Classificação do tipo, origem e localização das neoplasias neurológicas dos caninos encaminhados ao LRD/FV/UFPel e determinação do sexo, idade e raça dos animais acometidos.

\begin{tabular}{|c|c|c|c|c|c|c|}
\hline Diagnóstico & $\mathbf{N}$ & Origem & Localização (n) & $\operatorname{Sexo}(n)$ & $\begin{array}{c}\text { Idade média } \\
\text { (anos) }\end{array}$ & Raça (n) \\
\hline Carcinoma de mama & 8 & $S$ & $\begin{array}{l}\text { ME (1) } \\
\text { MF (4) } \\
\text { TC (3) }\end{array}$ & $\mathrm{F}(8)$ & 10,8 & $\begin{array}{c}\text { SRD (2) } \\
\text { Collie (1) } \\
\text { Daschund (1) } \\
\text { Boxer (1) } \\
\text { Cocker (1) } \\
\text { FB (1) } \\
\text { PA (1) }\end{array}$ \\
\hline Meningioma & 7 & $\mathrm{P}$ & $\mathrm{TC}(7)$ & $\begin{array}{l}F(5) \\
M(2)\end{array}$ & 10,7 & $\begin{array}{c}\text { SRD (3) } \\
\text { Labrador (1) } \\
\text { Husky (1) } \\
\text { Bulldog (1) } \\
\text { Rottweiler (1) }\end{array}$ \\
\hline Hemangiossarcoma & 5 & $\mathrm{~S}$ & $\begin{array}{l}\text { MF (2) } \\
\text { NI (3) }\end{array}$ & $\begin{array}{l}\mathrm{F}(2) \\
\mathrm{M}(3)\end{array}$ & 11 & $\begin{array}{c}\text { Daschund (2) } \\
\text { Cocker (1) } \\
\text { SRD (2) }\end{array}$ \\
\hline Linfoma & 5 & $\mathrm{~S}$ & $\begin{array}{c}\mathrm{C}(2) \\
\mathrm{ME}(1) \\
\mathrm{TC}(1) \\
\mathrm{NI}(1) \\
\end{array}$ & $\begin{array}{l}F(3) \\
M(2)\end{array}$ & 7,3 & $\begin{array}{c}\text { SRD (2) } \\
\text { Cocker (1) } \\
\text { Pug (1) } \\
\text { Rottweiler (1) }\end{array}$ \\
\hline Astrocitoma gemistocítico & 2 & $P$ & TC (2) & $\mathrm{M}(2)$ & 6 & $\begin{array}{c}\text { Cocker (1) } \\
\text { Yorkshire (1) }\end{array}$ \\
\hline Carcinoma broncoalveolar & 2 & $\mathrm{~S}$ & $\begin{array}{c}\text { C (1) } \\
\mathrm{ME}(1)\end{array}$ & $F(2)$ & 12,5 & $\begin{array}{c}\text { Rottweiler (1) } \\
\text { Boxer (1) }\end{array}$ \\
\hline Carcinossarcoma de mama & 1 & $\mathrm{~S}$ & $\mathrm{TC}(1)$ & $\mathrm{F}(1)$ & 13 & SRD (1) \\
\hline CGH & 1 & $\mathrm{~S}$ & ME (1) & $\mathrm{M}(1)$ & 6 & Cocker (1) \\
\hline Leucemia mielóide aguda & 1 & $\mathrm{~S}$ & MF (1) & $\mathrm{M}(1)$ & 8 & SRD (1) \\
\hline Neuroblastoma olfatório & 1 & $\mathrm{P}$ & $\mathrm{TC}(1)$ & $\mathrm{M}(1)$ & 13 & SRD (1) \\
\hline OSE & 1 & $\mathrm{~S}$ & $\mathrm{TC}(1)$ & $\mathrm{M}(1)$ & 10 & Cimarrom (1) \\
\hline TVT extragenital & 1 & $\mathrm{~S}$ & $\mathrm{TC}(1)$ & $\mathrm{F}(1)$ & 6 & SRD (1) \\
\hline
\end{tabular}

$\mathrm{P}=$ Primária, $\mathrm{S}=$ secundária, $\mathrm{M}=$ macho, $\mathrm{F}=$ fêmea, $\mathrm{SRD}=$ sem raça definida, $\mathrm{TC}=$ tálamo-córtex, $\mathrm{C}=$ cerebelo, $\mathrm{ME}=$ medula espinhal, $\mathrm{MF}=$ multifocal, $\mathrm{CGH}=$ carcinoma de glândula hepatóide, OSE = osteossarcoma extra-esquelético, TVT $=$ tumor venéreo transmissível, FB = Fila Brasileiro, PA = Pastor Alemães, SRD = sem raça definida. Fonte: Autores.

\section{Discussão}

No presente estudo, as neoplasias representaram 7,6\% das doenças neurológicas diagnosticadas em caninos e 4,9\% em felinos. Segundo Frade et al. (2014) e Chaves et al. (2018b) as neoplasias são a terceira causa mais frequente de doença neurológica nestas espécies e correspondem a 5,9\% e 10,3\% das patologias do SNC em caninos e felinos, respectivamente. Relaciona-se a baixa frequência de neoplasias neurológicas nos felinos deste estudo a menor casuística de animais desta espécie encaminhados ao LRD/FV/UFPel. 
A idade média dos animais foi de 9,5 anos para os cães e 7,3 anos para os gatos, semelhante ao observado por outros autores (Troxel et al., 2003; Tomek et al., 2006; Song et al., 2013; Chaves et al., 2018a; Chaves et al., 2018b). Nos caninos, as neoplasias ocorreram em 100\% dos casos em animais com idade igual ou superior a cinco anos, corroborando com Santos et al. (2012). A ocorrência de neoplasia neurológica em animais jovens é rara e ocorre principalmente em casos de tumores de origem embrionária (Horta et al., 2013). Neste trabalho o único diagnóstico em animal jovem foi um linfoma em felino, sendo este um neoplasma de maior ocorrência em gatos de até 24 meses (Mandara et al., 2016; Mello et al., 2019).

Quanto ao sexo, as fêmeas foram as mais acometidas, representando 62,9\% dos casos em caninos e 83,3\% em felinos. A maior ocorrência de neoplasias neurológicas em cadelas, também, foi observada por Chaves et al. (2018a), porém com menor representação (55\%). Já nos felinos, Troxel et al. (2003) e Tomek et al. (2006) descreveram um maior acometimento de machos do que de fêmeas. Em ambas as espécies não há confirmação de predisposição sexual na ocorrência de neoplasias neurológicas (Troxel et al., 2003; Snyder et al., 2006; Chaves et al., 2018a), porém os meningiomas são observados com maior frequência em fêmeas (PLATT et al., 2006; CHAVES et al., 2018a), assim como no presente trabalho. Em humanos a maior ocorrência de meningiomas em mulheres está associado a presença de receptores de progesterona no tumor (Hsu et al., 1997; Marcasso et al., 2015), entretanto, em animais a relação dos hormônios femininos na ocorrência deste neoplasma não foi comprovada (Marcasso et al., 2015). Neste trabalho, em ambas as espécies, as neoplasias mais frequentes foram o meningioma e as metástases de tumores de mama, o que pode justificar o maior número de casos em fêmeas.

Em relação a raça, nos caninos a maior frequência foi de CRD, corroborando com Santos et al. (2012) e diferindo de Chaves et al. (2018a) e Song et al. (2013), que observaram uma maior ocorrência nos SRD. Segundo a literatura os cães da raça Boxer apresentam uma maior predisposição para a ocorrência de neoplasias neurológicas (Santos et al., 2012; Song et al., 2013; Chaves et al., 2018a), o que não foi observado neste estudo, visto que apenas 2/35 cães eram desta raça. Embora o Boxer seja considerado uma raça predisposta a desenvolver neoplasias neurológicas, estudos epidemiológicos podem apresentar divergências em relação a raça mais acometida, pois as características populacionais variam de acordo com a região estudada. Nos felinos 4/6 casos ocorreram em animais SRD e 2/6 em gatos persa, diferindo dos achados de Troxel et al. (2003) e Tomek et al. (2006), onde a maior ocorrência foi em gatos da raça pelo curto doméstico.

Os sinais neurológicos, apresentados pelos animais deste estudo, foram similares aos descritos na literatura em casos de neoplasias no SNC de caninos e felinos (Troxel et al., 2003; Snyder et al., 2006; Marioni-Henry et al., 2008; Snyder et al., 2008; Santos et al., 2012; Chaves et al., 2018a; Andrade et al., 2019). Embora convulsão e alteração do comportamento possam ser indicativos precoces de neoplasia no SNC (Horta et al., 2013) em alguns casos podem não ser evidenciadas manifestações neurológicas (Troxel et al., 2003; Sapin et al., 2019). No presente estudo, 34,3\% dos caninos e 50\% dos felinos não apresentaram sinais neurológicos, sendo o diagnóstico realizado apenas no exame post mortem. Dessa forma, salienta-se a importância da necropsia para determinar a frequência de neoplasias neurológicas em caninos e felinos.

Nos caninos a região tálamo-córtex foi a mais acometida (17/35; 48,6\%), sendo o meningioma o neoplasma de maior ocorrência nesta região. Resultados semelhantes foram observados em trabalhos anteriores (Snyder et al., 2006; Santos et al., 2012; Higgins et al., 2017; Chaves et al., 2018a). As lesões multifocais representaram a segunda maior casuística (7/35; 20\%) nos cães, corroborando com Chaves et al. (2018a). Entretanto, no presente trabalho as lesões multifocais foram mais frequentes nos casos de metástase de carcinoma de mama (4/7) e de hemangiossarcoma (2/7), diferindo de Chaves et al. (2018a) onde os tumores primários representaram o maior número de casos. A distribuição multifocal de neoplasias neurológicas é mais frequente em tumores secundários, devido a ocorrência de metástases por via hematógena, com posterior disseminação pelo líquor (Horta et al., 2013). Nos felinos a região mais afetada foi a medula espinhal (3/6; 50\%), sendo os três casos decorrentes de neoplasias secundárias, dois por metástase (linfoma e carcinoma tubular de mama) e um por expansão tumoral (fibrossarcoma). Nesta espécie estima-se que apenas 18,7\% das neoplasias neurológicas ocorrem na medula espinhal (Chaves 
et al., 2018b), sendo o linfoma o neoplasma mais frequente, representando 38,8\% dos tumores medulares em gatos (MarioniHenry et al., 2008; Mandara et al., 2016; Mello et al., 2019). Apesar do maior índice de neoplasias na medula espinhal, neste estudo, não podemos definir este como o principal sítio de neoplasias neurológicas em felinos, tendo em vista o baixo número de casos desta espécie encaminhados ao LRD/FV/UFPel.

Em ambas as espécies, as neoplasias secundárias foram as de maior ocorrência, representando 70,6\% dos casos em caninos e 83,3\% nos felinos corroborando com Heck et al. (2018) porém diferindo de outros estudos (Troxel et al., 2003; Santos et al., 2012; Spitzbarth et al., 2017; Chaves et al., 2018a; Kishimoto et al., 2020). Assim como observado por Heck et al. (2018) a neoplasia secundária de maior ocorrência foi o carcinoma mamário. O principal mecanismo de disseminação dos neoplasmas, observado nesse estudo, foi a metástase, sendo apenas um caso devido a tumor infiltrativo. Diferindo este resultado de outros autores que descreveram a proporção de neoplasias secundárias infiltrativas e metastáticas como semelhantes (Santos et al., 2012; Chaves et al., 2018a). Dentre os tumores secundários os carcinomas de mama foram os mais frequentes, em ambas as espécies e, em 100\% destes casos havia, também, metástase no pulmão. Nos humanos o índice de metástase cerebral nos casos de tumores de mama é alto, observado em 30\% dos casos, sendo este risco aumentado quando já ocorreram metástases pulmonares (Azim et al., 2017). Em cães e gatos os linfonodos regionais e pulmão são os principais sítios de metástase dos tumores de mama, já as metástases cerebrais são menos frequentes, ocorrendo normalmente em estágios finais da doença (Castro et al., 2017).

\section{Conclusão}

Com base nos resultados descritos neste trabalho, podemos concluir que as neoplasias neurológicas secundárias foram as mais frequentes em caninos e felinos. Em ambas as espécies, metástase de carcinoma mamário foi o tumor neurológico secundário mais frequente, sendo observado em $100 \%$ dos casos, também, metástase no pulmão. Pode-se concluir ainda que em muitos casos de neoplasia no SNC os sinais clínicos neurológicos podem estar ausentes, sendo o exame post mortem fundamental para a análise de ocorrência destes tumores. Dessa forma, reforça-se a importância de realizar estudos retrospectivos de neoplasias neurológicas em caninos e felinos, buscando assim estabelecer os principais aspectos epidemiológicos e determinar a real prevalência destas neoplasias em diferentes regiões.

\section{Referências}

Andrade, T. M., Caramalac, S. M., Pupin, R. C., Babo-Terra V. J. \& Palumbo, M. I. P. (2019). Ependimoma anaplásico em cão. Acta Scientiae Veterinariae, 47(Suppl 1): 389-342.

Azim, H. A., Abdel-Malek, R. \& Kassem, L. (2017). Predicting brain metastasis in breast cancer patients: stage versus biology. Clinical Breast Cancer, 18(2): $187-195$.

Belluco, S., Avallone, G., Di Palma, S., Rasotto, R. \& Oevermann, A. (2019). Inter- and intraobserver agreement of canine and feline nervous system tumors. Veterinary Pathology, 56(3):342-349.

Besalti, O., Caliskan, M., Can, P., Vural, S. A., Algin, O. \& Ahlat, O. (2016). Imaging and surgical outcomes of spinal tumors in 18 dogs and one cat. Journal of Veterinary Science, 17(2):225-234.

Castro, M. R., Gouveia, B. A., Silva, M. C. B., Medeiros-Ronchi, A. A. \& Bastos, J. E. D. (2017). Metástase de carcinoma mamário em sistema nervoso central de cadela. Enciclopédia Biosfera, 14(26): 438-445.

Cantile, C. \& Youssef, S. (2016). Nervous System. In: Jubb, K. V. F., Kennedy, J. P. \& Palmer's Pathology of Domestic Animals. St. Louis: Elsevier, 250406.

Chaves, R. O., Feranti, J. P. S., Copat, B., Ripplinger, A., França, R. T., Kommers, G. D., Fighera, R. A. \& Mazzanti, A. (2018a). Neoplasias encefálicas em 40 cães: aspectos clínico-epidemiológicos e patológicos. Pesquisa Veterinária Brasileira, 38(4): 734-740.

Chaves, R. O., Togni, M., Copat, B., Feranti, J. P. S., Silva, A. P., França, R. T., Fighera, R. A. \& Mazzanti, A. (2018b). Doenças neurológicas em gatos: 155 casos. Pesquisa Veterinária Brasileira, 38(1): 107-112. 
Coates, J. R. \& Johnson, G. C. (2010). Nervous system neoplasia. In: Henry, C. J., Higginbothan, M. L. (Eds). Cancer management in small animal practice. Missouri: Sauders, p. 186-194.

Frade, M. T. S., Ferreira, J. S., Nascimento, M. J. R., Aquino, V. V. F., Macêdo, I. L., Carneiro, R. S., Souza, A. P. \& Dantas, A. F. M. (2018). Doenças do sistema nervoso central em cães. Pesquisa Veterinária Brasileira, 38(5): 935-948.

Fraga, C. A. T., Costa, T. C., Headley, S. A. \& Gomes, L. A. (2014). Alterações neurológicas associadas a tumor cerebral. PUBVET, 8(23): $2806-2887$.

Frank, L., Burigk, L., Lehmbecker, A., Wohlsein, P., Schütter, A., Meyerhoff, N., Tipold, A. \& Nessler, J. (2020) Meningioma and associated cerebral infarction in three dogs. BMC Veterinary Research, 16(1):177.

Heck, L. C., Cony, F. G., Bianchi, M. V., Driemeier, D., Sonne, L. \& Pavarini, S. P. (2018). Pathological features of 78 metastatic or multicentric neoplasms involving the central nervous system in dogs. Pesquisa Veterinária Brasileira, 38(10):1989-1998.

Higgins, R. J., Bollen, A. W., Dickinson, P. J. \& Sisó-Lionch, S. (2017). Tumors of the nervous system. In: Meuten, D. J. (Ed). Tumors of Domestic Animals. 5ed. Iowa: State Press, 834-891.

Horta, R. S, Martins, B. C., Lavalle, G. E., Costa, M. P. \& Araújo, R. B. (2013). Neoplasias intracranianas em pequenos animais - revisão de literatura. Acta Veterinaria Brasilica, 7(4): 272-281.

Hsu, D. W., Efird, J. T. \& Hedley-Whyte, E. T. (1997). Progesterone and estrogen receptors in meningiomas: prognostic considerations. Journal of Neurosurgery, 86(1): 113-120.

Kishimoto, T. E., Uchida, K., Chambers, J. K., Kok, M. K., Filho, N. V., Shiga, T., Hirabayashi, M., Ushio, N. \& Nakayama H. (2020). A retrospective survey on canine intracranial tumors between 2007 and 2017. The Journal of Veterinary Medical Science, 82(1):77-83.

Mandara, M. T., Motta, L., \& Calò, P. (2016). Distribution of feline lymphoma in the central and peripheral nervous systems. The Veterinary Journal, 216:109-116.

Marcasso, R. A., Moreira, J. R., Valentim, L. G., Arias, M. V. B. \& Bracarense, A. P. F. R. L. (2015). Meningiomas em cães: aspectos clínicos, histopatológicos e imuno-histoquímicos. Pesquisa Veterinária Brasileira, 35(10): 844-852.

Marioni-Henry, K., Winkle, T. J. V., Smith, S. H. \& Vite, C. H. (2008). Tumors affecting the spinal cord of cats: 85 cases (1980-2005). Journal of the American Veterinary Medical Association, 232(2): 237-243.

Mcentee, M. C. \& Dewey, C. W. (2013). Tumors of the nervous system. In: Withrow, S. J., Vail, D. M., Page, R. L. (Eds). Small Animal Clinical Oncology. Philadelphia: Saunders, p. 583-596.

Mello, L. S., Leite-Filho, R. V., Panziera, W., Bandinelli, M. B., Sonne L., Driemeier, D. \& Pavarini, S. P. (2019). Feline lymphoma in the nervous system: pathological, immunohistochemical, and etiological aspects in 16 cats. Pesquisa Veterinária Brasileira, 39(6):393-401.

Platt, S. R., Scase, T. J., Adams, V., Wieczorek, L., Miller, J., Adamo, F. \& Long, S. (2006).Vascular endothelial growth factor expression in canine intracranial meningiomas and association with patient survival. Journal of Veterinary Internal Medicine, 20(3): 663-668.

Prates, K. S., Bianchi, M. V., Mello, L. S., Matesco, V. C., Lorenzo, C., Driemeier, D. \& Pavarini, S. P. (2018). Spinal cord anaplastic meningioma with extra-neural metastasis in a cat. Ciência Rural, 48: e20180063.

Santos, R. P., Fighera, R. A., Beckmann, D. V., Brum, J. S., Ripplinger, A., Neto, D. P., Baumhardt, R. \& Mazzanti, A. (2012). Neoplasmas envolvendo o sistema nervoso central de cães: 26 casos (2003-2011). Pesquisa Veterinária Brasileira, 32(2): 153-158.

Sapin, C. F., Fernandes, C. G., Nobre, M. O. \& Grecco, F. B. (2019). Neoplasmas encefálicos em cães idosos. Acta Scientiae Veterinariae, $47(1)$ : 384.

Snyder, J. M., Lipitz, L., Skorupski, K. A., Shofer, F. S. \& Winkle, T. J. V. (2008). Secondary intracranial neoplasia in the dog: 177 cases (1986-2003). Journal of Veterinary Internal Medicine, 22(1):172-177.

Snyder, J. M., Shofer, F. S., Winkle, T. J. V. \& Massicotte, C. (2006). Canine intracranial primary neoplasia: 173 cases (1986-2003). Journal of Veterinary Internal Medicine, 20(3): 669-675.

Song, R. B., Vite, C. H., Bradley, C. W. \& Cross, J. R. (2013). Postmortem Evaluation of 435 Cases of intracranial neoplasia in dogs and relationship of neoplasm with breed, age, and body weight. Journal of Veterinary Internal Medicine, 27(5): 1143-1152.

Spitzbarth, I., Heinrich, F., Herder, V., Recker, T., Wohlsein, P. \& Baumgärtner, W. (2017). Canine central nervous system neoplasm phenotyping using tissue microarray technique. Veterinary Pathology, 54(3): 369-379.

Tomek, A., Cizinauskas, S., Doherr, M., Gandini, G. \& Jaggy, A. (2006). Intracranial neoplasia in 61 cats: localisation, tumour types and seizure patterns. Journal of Feline Medicine and Surgery, 8(4): 243-253.

Troxel, M. T., Vite, C. H., Winkle, T. J. V., Newton, A. L., Tiches, D., Dayrell-Hart, B., Kapatkin, A. S., Shofer, F. S. \& Steinberg, S. A. (2003). Feline intracranial neoplasia: retrospective review of 160 cases (1985-2001). Journal of Veterinary Internal Medicine, 17:850-859.

Zamboni, R., Alberti, T. S., Schied, H. V., Bermann, C. S., Brunner, C. B., Venancio, F. R., Arantes, E. M. J., Sallis, E. S. V. \& Raffi, M. B. (2020). Meningioma microcístico com metástase pulmonar em canino: relato de caso. Arquivo Brasileiro de Medicina Veterinária e Zootecnia, 72(3): 794-798. 